\title{
ÍNDICE DE MASA CORPORAL, CIRCUNFERENCIA DE CINTURA Y DIABETES EN ADULTOS DEL ESTADO DE MÉXICO.
}

\author{
BODY MASS INDEX, WAIST CIRCUMFERENCE AND DIABETES IN ADULTS OF THE STATE OF MEXICO.
}

Lozano Keymolen Daniel*, Gaxiola Robles Linares Sergio Cuauhtémoc*.

Citation: Lozano Keymolen D., Gaxiola Robles Linares S.C. (2020) Índice de masa corporal, circunferencia de cintura y diabetes en adultos del Estado de México. Revista Salud Pública y Nutrición, 19 (1), 10-22.

Editor: Esteban G. Ramos Peña, Dr. CS., Universidad Autónoma de Nuevo León, Facultad de Salud Pública y Nutrición, Monterrey Nuevo León, México. Copyright: (C2020 Lozano Keymolen D. et al. This is an open-access article distributed under the terms of Creative Commons Attribution License [CC BY 4.0], which permits unrestricted use, distribution, and reproduction in any medium, provided the original author and source are credited.

Competing interests: The authors have declared that no competing interests exist.

DOI: https://doi.org/10.29105/respyn19.1-2

Recibido: 29 de enero 2020;

Aceptado: 20 de abril 2020

Email: daniel.lozkey@gmail.com

*Universidad Autónoma del Estado de México, Centro de Investigación y Estudios Avanzados de la Población, México. 


\title{
ÍNDICE DE MASA CORPORAL, CIRCUNFERENCIA DE CINTURA Y DIABETES EN ADULTOS DEL ESTADO DE MÉXICO.
}

\author{
Lozano Keymolen Daniel*, Gaxiola Robles Linares Sergio Cuauhtémoc*. \\ Universidad Autónoma del Estado de México, México.
}

\section{RESUMEN}

Introducción. La diabetes y la obesidad son importantes problemas de salud pública en el Estado de México. Objetivo: Determinar la precisión diagnóstica y los puntos de corte óptimos del índice de masa corporal y de la circunferencia de cintura sobre la diabetes, así como conocer la asociación de ambos indicadores con la enfermedad según los puntos de corte definidos. Material y Método: Los datos son de la Encuesta Nacional de Salud y Nutrición 2012 para los adultos del Estado de México. Se analizaron 969 observaciones de adultos con datos completos en las variables de análisis. Se estimaron curvas ROC para determinar la precisión diagnóstica y el valor del punto de corte del índice de masa corporal y de la circunferencia de la cintura sobre la diabetes. Definidos los puntos de corte se estimaron regresiones logísticas para ambos indicadores ajustando por diversas variables. Resultados: Se obtuvieron mayores valores de sensibilidad y especificidad para la circunferencia de la cintura: una circunferencia de cintura $\geq 94.950$ en hombres $y \geq 91.050$ en mujeres incrementa la propensión a diabetes entre 2.4 y 2.6 veces. Conclusiones: En la muestra analizada, la circunferencia de la cintura es mejor predictor de la diabetes que el índice de masa corporal.

Palabras Clave: Diabetes, circunferencia de cintura, índice de masa corporal, curvas ROC.

\section{ABSTRACT}

Introduction: Diabetes and obesity are important public health problems in the State of Mexico. Objective: To determine the diagnostic accuracy and optimal cut-off points of the body mass index and waist circumference on diabetes, as well as to know the association of both indicators with the disease according to the defined cut-off points. Material and method: The data are from the National Health and Nutrition Survey 2012 for adults in the State of Mexico. 969 observations of adults with complete data in the analysis variables were analyzed. ROC curves were calculated to determine the diagnostic accuracy and the cut-off value of the body mass index and waist circumference over diabetes. Once the cut-off points were defined, logistic regressions were estimated for both indicators, adjusting for various variables. Results: Higher sensitivity and specificity values for waist circumference were obtained: a waist circumference $\geq 94,950$ in men and $\geq 91,050$ in women increases the propensity to diabetes between 2.4 and 2.6 times. Conclusions: In the analyzed sample, waist circumference is a better predictor of diabetes than the body mass index.

Key words: Waist circumference, body mass index, ROC curves. 


\section{Introducción}

La diabetes se define como una enfermedad crónica compleja que requiere atención médica continúa en conjunto con estrategias de reducción de riesgos multifactoriales más allá de solo el control glucémico (American Diabetes Association (ADA), 2020a). Las complicaciones de la diabetes se expresan en daños en los ojos, complicaciones renales, ataques cardiacos o discapacidades y amputaciones (Organización Mundial de la Salud (OMS), 2018). A nivel mundial, la diabetes es una de las causas principales de morbilidad y de mortalidad. A nivel global, se estimó que, en el 2014, el $8.5 \%$ de las personas con 18 años y más tienen diabetes, causando 1.6 millones de defunciones en el 2016 (OMS, 2018).

Los factores de riesgo para el desarrollo de diabetes refieren, por un lado, a los procesos biológicos de la enfermedad (ADA, 2020b) y, por otro lado, a factores sociodemográficos, los cuales pueden clasificarse en dos tipos: a) factores modificables: como la inactividad física, la ingesta elevada de grasas y azúcares o la obesidad, así como variables socioeconómicas como la pobreza o la disponibilidad de servicios de salud (den Braver et al., 2018; Hernández-Ávila, Gutiérrez y Reynoso-Noverón, 2013; Kutbi, Mosli, Alhasan y Mosli, 2018); b) factores no modificables: el sexo y la edad, pues tanto en las mujeres como en la edad avanzada se presentan mecanismos que incentivan la diabetes, asimismo la genética o la presencia de antecedentes parentales de la enfermedad, entre otros (ADA, 2020b; Rull et al., 2005).

Sin embargo, la obesidad, una acumulación anormal o excesiva de grasa corporal, es considerada el principal factor de riesgo para la diabetes, dado que la primera puede ser resultado de un conglomerado de variables predisponentes para la diabetes. Diversas investigaciones han asociado a la obesidad con el riesgo de desarrollar diabetes, particularmente con la de tipo 2 (ADA, 2020b; Estampador y Franks, 2018; Al-Goblan, Al-Alfi y Kahn, 2014). Incluso, se estimó que hasta $80.0 \%$ de los casos de diabetes tipo 2 en adultos se relacionan con la obesidad (Gatineau et al., 2014). La estimación en adultos mexicanos de 50 años o más indicó que la propensión a tener diabetes fue tres veces mayor entre quienes presentaban obesidad (Pinto y Beltrán-Sánchez, 2015).
Existe una estrecha relación de la diabetes con la obesidad, pues el riesgo de desarrollar diabetes incrementa conforme lo hace el exceso de peso corporal. Investigaciones sugieren que, a lo largo de un periodo de 10 años, por cada kilogramo $(\mathrm{kg}) \mathrm{de}$ peso acumulado, el riesgo de desarrollar diabetes incrementa en un $49.0 \% \mathrm{y}$, por el contrario, quienes pierden peso, el riesgo disminuye (Aucott, 2008). Además, se sabe que en individuos con obesidad los procesos relacionados con la resistencia a la insulina se incrementan; particularmente, las células del páncreas se dañan, incentivando la falta de control de la glucosa en sangre, lo que aumenta la incidencia de diabetes tipo 2 (ADA, 2020b; Kahn, Hull y Utzschneider, 2006). De hecho, la diabetes tipo 2 en personas con obesidad es común, al grado de considerárseles comórbidas, ya que son enfermedades que suelen aparecer conjuntamente (Jarolimova, Tagoni y Stern, 2013). Sin embargo, otros factores como el tiempo de evolución de la obesidad o la distribución de la grasa corporal son elementos que también inciden en el desarrollo de diabetes (Aucott, 2008; González-Villalpando, Dávila-Cervantes, Zamora-Macorra, Trejo-Valdivia y González-Villalpando, 2014).

Aunque el índice de masa corporal (IMC) es el indicador de obesidad más empleado tanto en estudios de salud pública como de clínica, existe evidencia de que el IMC no discrimina la distribución de la grasa corporal (Liu, Tong, Tong, Lu y Qin, 2011; Kim et al., 2019). De ahí que en diversas investigaciones se ha propuesto que el indicador de la circunferencia de la cintura (CC) es un mejor predictor de la diabetes que el IMC, dado que el primero permite determinar la distribución de la grasa corporal e incluso es predictivo de comorbilidades y de la mortalidad (Sosenko et al.,1993; Janssen, Katzmarzyk y Ross, 2004; Cerhan et al., 2014; Kim et al., 2019), pues las personas con elevada acumulación de grasa en la cintura tienen mayor riesgo de daño en la producción de insulina (Ye, 2013; Gómez-Ambrosi et al., 2011; Haffner, 1998). Además, algunas investigaciones han determinado que la $\mathrm{CC}$ es un mejor indicador del riesgo de diabetes, incluso entre las personas con un IMC en las categorías de bajo peso o normal (Feller, Boeing y Pischon, 2010). Sin embargo, la evidencia no es concluyente dado que una meta-análisis de 32 estudios indicó que tanto el IMC como la CC serían predictores consistentes de la diabetes (Vázquez, 
Duval, Jacobs y Silventoinen, 2007), por lo cual podrían existir diferencias entre poblaciones (Qiao y Nyamdorj, 2010).

Entre las causas principales de morbilidad y de mortalidad de la población mexicana se encuentra la diabetes, pues según la Encuesta Nacional de Salud y Nutrición 2018 (ENSANUT 2018), entre adultos mexicanos (20 años y más), la prevalencia de diabetes por autoreporte es $10.3 \%$ (Instituto Nacional de Salud Pública (INSP), 2019a), lo que es un incremento respecto de las prevalencias con la ENSANUT MC de 2016, que fue $9.4 \%$, y $9.2 \%$ con la ENSANUT 2012 (Rojas-Martínez et al., 2018). De manera similar, en México la población adulta registra una de las prevalencias de obesidad más elevadas en el mundo (Chooi, Din y Magkos, 2019). La ENSANUT 2018 arrojó que $36.1 \%$ de los mexicanos adultos tenía obesidad (INSP, 2019a), lo que representó un aumento respecto de la ENSANUT MC cuya prevalencia fue $32.8 \%$, y de $32.4 \%$ en la ENSANUT 2012.

Datos como los anteriores indican que la diabetes y la obesidad son importantes problemas de salud pública en México. Sin embargo, la asociación de estas dos condiciones crónicas, en niveles menores de desagregación geográfica a la nacional, son poco conocidas (Hernández-Ávila et al., 2013; RojasMartínez et al., 2018). Respecto de lo anterior, este artículo realiza una investigación para la población del Estado de México en la que la diabetes es una de las principales causas de enfermedad y de muerte ya que en 2018 la enfermedad se situaba entre las 10 causas principales de morbilidad (Secretaria de Salud, 2020). Con datos de la ENSANUT 2012 se calculó que el porcentaje de mexiquenses con diabetes fue $10.5 \%$, lo que representa un valor por arriba del promedio nacional, que fue $9.2 \%$ para ese mismo año (Hernández-Ávila et al., 2013). En cuanto a la mortalidad, en el mismo año de 2012, la diabetes fue la causa principal de muerte en la población mexiquense ocasionando cerca del $17.07 \%$ de las defunciones (SINAVE/DGE/SALUD, 2020).

Analizar la asociación que tiene la diabetes con la obesidad en una población con una mayor prevalencia que la nacional podría informar de una de las relaciones más trascendentales para el perfil de salud y de mortalidad entre la población mexiquense, pues además es la entidad más poblada del país con cerca de 16.2 millones habitantes en el 2020 (Consejo Nacional de Población (CONAPO), 2018). Entonces, el objetivo de esta investigación se plantea en dos etapas. En primer lugar, determinar la capacidad diagnóstica y los puntos de corte del IMC y de la CC en la probabilidad de presentar diabetes por sexo. En segundo lugar, conocer la asociación de ambos indicadores con la diabetes entre adultos del Estado de México según los puntos de corte definidos. En este sentido, se hipotetiza que tanto el IMC como la CC son indicadores predictivos de la diabetes.

\section{Material y Método}

Fuente de datos y muestra

Los datos se obtuvieron de la ENSANUT 2012, una encuesta transversal con diseño muestral polietápico y estratificado que permite obtener representatividad de la población mexicana, a nivel nacional, de los ámbitos rurales y urbanos, así como de las 32 entidades federativas de México. Se empleó la ENSANUT 2012 porque si bien existe la ENSANUT 2018, ésta no tiene representatividad a nivel entidad federativa de las componentes de nutrición (INSP, 2019b), y no se usó tampoco la ENSANUT MC, pues la encuesta no es representativa a nivel estatal (Romero-Martínez et al., 2017).

La muestra analítica se integró por los adultos (20 años de edad o más) residentes en el Estado de México al momento de la ENSANUT 2012. Originalmente esta encuesta tuvo una muestra constituida por 1,443 adultos, representativos de 9,523,317 personas en el 2012 (INSP, 2013). Sin embargo, para este estudio solo se analizaron los casos con información completa en las variables de interés, por lo que el tamaño de la muestra analizada fue de 969 observaciones (55.4\% mujeres) que, aplicando el diseño complejo de la ENSANUT 2012, en dicho año representaron a 6,069,570 personas.

\section{Consideraciones éticas}

La ENSANUT 2012 es una encuesta de uso público y fue aprobaba por las Comisiones de Ética, Investigación y Bioseguridad del Instituto Nacional de Salud Pública (INSP) de México. Además, la ENSANUT 2012 obtuvo consentimiento informado de los entrevistados para el manejo de sus datos (INSP, 2013). Esta investigación se desarrolló considerando que no existe contacto con seres 
humanos al utilizar datos provenientes de una fuente secundaria.

Variables bajo análisis

Diabetes. Fue la variable dependiente de esta investigación. Esta variable se obtuvo con el reactivo (¿Algún médico le ha dicho que tiene diabetes o alta el azúcar en la sangre?), constituyendo una variable dicotómica $(1=$ diabetes, $0=$ otro caso). Así, se trata de una definición basada en autoreporte que no se limita a la diabetes tipo 2 si bien la evidencia indica que entre el 90-95\% de los casos de diabetes son del tipo 2 (ADA, 2020b). Sin embargo, existe evidencia de que el autoreporte de diabetes es un indicador consistente para determinar la presencia de la enfermedad en el nivel poblacional (Yuan et al., 2015).

Según los objetivos de la investigación se plantean dos variables independientes: IMC y CC. Estas variables se obtuvieron mediante mediciones antropométricas de peso, altura y circunferencia de cintura que en la ENSANUT 2012 fueron obtenidas con equipos y técnicas estandarizadas y levantadas por personal capacitado (Romero-Martínez et al., 2013).

Índice de masa corporal (IMC). El IMC es un indicador de peso corporal de extenso uso en estudios poblacionales y de fácil calculo. El IMC se obtuvo de las mediciones antropométricas de peso y talla disponibles en la ENSANUT 2012. El peso se obtuvo con una precisión de $100 \mathrm{~g}$ con una balanza electrónica y la talla con un estadiómetro con precisión de 1mm (Barquera et al., 2013). La variable para el peso se obtuvo en $\mathrm{kg}$ por lo cual no fue necesario procesar los datos, caso contrario al de la variable talla, ya que esta variable se recopiló en $\mathrm{cm}$ por lo que se dividió entre 100 para obtener los valores en metros. Obtenidas las variables de peso y talla, el IMC se calculó como el cociente que resulta de dividir el peso por el cuadrado de la talla $(\mathrm{kg} / \mathrm{m} 2)$. Para los fines de esta investigación, el IMC se consideró de forma continua (Feller et al., 2010; Janssen et al., 2004). En esta estimación no se tomó en cuenta a las mujeres embarazadas ni a aquellos casos con IMC mayores a $58 \mathrm{~kg} / \mathrm{m} 2$.

Circunferencia de la cintura. Para determinar el exceso de grasa en la cintura se emplearon las mediciones de la circunferencia de la cintura (CC) disponibles en la ENSANUT 2012. Las mediciones de la CC se obtuvieron en centímetros midiendo el punto medio entre la parte inferior de las costillas y la parte superior de la cresta iliaca (Albrecht, Barquera y Popkin, 2014) y solo se consideraron como válidos los valores en el intervalo $50-180 \mathrm{~cm}$. Al igual que el IMC, la CC se utilizó de forma continua (Feller et al., 2010; Janssen et al., 2004).

Aunque existen una serie de variables que se considera median la asociación entre la diabetes y la obesidad, y que a la vez sirven como predictores de cada condición, la presente investigación se enfoca en seis, que son: edad, antecedentes parentales de diabetes, pertenencia indígena, consumo de tabaco, escolaridad y afiliación a servicios de salud.

La edad es uno de los predictores que relacionan a la diabetes con la obesidad. Específicamente se ha encontrado que el riesgo de desarrollar diabetes incrementa con la edad, como resultado de una mayor exposición a los factores de riesgo conocidos, así como por el desgaste de los órganos que acompañan al envejecimiento (ADA, 2020a; Kirkman et al., 2012). Esta variable quedó definida en forma continua.

Antecedentes parentales de diabetes. Se retomaron dos reactivos de la ENSANUT 2012: ¿Su padre/madre tiene o tuvo diabetes o azúcar alta en la sangre? La variable fue codificada como dicotómica ( 1 = si del entrevistado la madre o el padre (o ambos) tenían o habían tenido diabetes y $0=$ otro caso).

Pertenencia indígena. Diversas investigaciones han mostrado diferencias existentes al utilizar el IMC o la CC como indicadores de obesidad entre diversos grupos raciales y de indígenas (Katzmarzyk et al., 2011; Yusuf et al., 2005). Para construir esta variable se utilizó el reactivo ¿Usted habla alguna lengua indígena? La variable fue dicotómica $(1=$ pertenencia indígena, $0=$ otro caso).

Consumo de tabaco. En cuanto al consumo de tabaco, diversas investigaciones han postulado los efectos del tabaquismo sobre el peso corporal. Es decir, una parte considerable de los fumadores aumentan de peso después de dejar de fumar lo cual se relaciona con resultados adversos como la aparición de diabetes (Bush et al., 2016). Esta 
variable fue codificada categórica ( $1=$ actualmente fuma, $2=$ antes fumaba, $3=$ nunca ha fumado).

Escolaridad. Se emplea como una variable asociada en la cual existe evidencia de gradientes socioeconómicos para la obesidad y la diabetes (Cohen, Rai, Rehkopf y Abrams, 2013; Steele et al., 2017). En este sentido, se ha encontrado evidencia de que los individuos con menor escolaridad tienen mayor incidencia de la diabetes, particularmente entre las personas con obesidad (Shang et al., 2013). Para construir esta variable se retomaron dos reactivos de la ENSANUT 2012 que informan sobre el último grado y año escolar aprobados. La variable fue codificada como categórica $(1=0-5$ años, $2=6-9$ años, $3=10$ y más años).

Servicios de salud. Adicionalmente, en este estudio se incluyó la afiliación a servicios de salud, dado que existe evidencia de que ésta se asocia con un mayor diagnóstico de diabetes (Beltrán-Sánchez, DrumondAndrade y Riosmena, 2015). Además, se ha hipotetizado que las personas con diabetes que utilizan los servicios de salud pueden tener tal conocimiento de la enfermedad que puede verse afectada su asociación con la obesidad (Kutbi et al., 2018). Esta variable fue dicotómica (1= afiliación a servicios de salud por IMSS/ISSSTE/SEDENA/Pemex o consulta privada, $0=$ otro caso).

\section{Análisis de datos}

El análisis estadístico comprendió tres procedimientos generales los cuales se estratificaron por sexo dado que existe evidencia de diferencias tanto en la prevalencia de diabetes (Huebschmann et al., 2019) como de obesidad (Kanter y Caballero, 2012). En el primer procedimiento, se estimaron los estadísticos descriptivos, en forma de distribuciones porcentuales para las variables categóricas (IC 95\%) y para las variables continuas como la media $( \pm$ desviación estándar) y valores mínimos y máximos. En este procedimiento se utilizaron los ponderadores poblacionales de la ENSANUT 2012 (RomeroMartínez et al., 2013). Paralelamente y para apoyar la inferencia sobre los estimadores se presentan las distribuciones de las variables sin ponderar y pruebas de significancia chi-cuadrada $\left(\chi^{2}\right)$ para variables categóricas y t-Student para variables continuas, según corresponda.
En el segundo procedimiento se estimaron curvas ROC dado que estas permiten determinar la precisión diagnóstica y el valor óptimo del punto de corte del IMC o de la CC sobre la diabetes. Los puntos de corte se definieron siguiendo la propuesta de Liu (2012) en la cual se maximiza la sensibilidad y la especificidad del IMC y de la CC. Luego, para determinar diferencias en ambos indicadores se calcularon estadísticos $\chi 2$ entre las áreas bajo la curva $(\mathrm{ABC})$ de los indicadores del IMC y de la CC con lo cual se determina el mejor indicador (Cleves, 2002).

En un tercer procedimiento se desarrollaron modelos de regresión logística utilizando los puntos de corte definidos para el IMC y la CC por sexo. Con base en lo anterior, se definieron dos ecuaciones: primero utilizando al IMC (modelo IMC) como variable predictora y en una segunda ecuación se ajustaron los efectos de la regresión anterior. Un procedimiento similar se realizó empleando la CC como variable predictora (modelo CC). Es decir, se estimó un total de 8 regresiones (4 por sexo). Según este procedimiento, las ecuaciones que determinan la probabilidad de tener diabetes (pi) son: $p_{i}=$ $\frac{1}{1+e^{-\left(\beta_{0}+\beta_{1} X_{i}+\varepsilon_{i}\right.}}$, donde: $\beta 0=$ intercepto; $\beta 1=$ efecto del IMC o de la CC; $\varepsilon i$ = errores de estimación. Luego, las regresiones se ajustaron definiéndose, $p_{i}=\frac{1}{1+e^{-\left(\beta_{0}+\beta_{1} X_{i}+\beta_{2} X_{i}+\beta_{3} X_{i}+\beta_{4} X_{i}+\beta_{5} X_{i}+\beta_{6} X_{i}+\varepsilon_{i}\right.}}$,

donde: $\beta 0=$ intercepto; $\beta 1=$ efecto del IMC o de la $\mathrm{CC} ; \beta 2=$ efecto de la edad; $\beta 3=$ efecto de los antecedentes parentales de diabetes; $\beta 4=$ efecto de la pertenencia indígena; $\beta 5=$ efecto del consumo de tabaco; $\beta 6=$ efecto de los servicios de salud; $\beta 7=$ efecto de la escolaridad; $\mathcal{E} \mathrm{i}=$ errores de estimación. Los datos se analizaron con el software Stata 14.1@ (StataCorp, 2016).

\section{Resultados}

En la tabla 1 se presenta la distribución porcentual de las variables de interés según los ponderadores de la ENSANUT 2012. La prevalencia ponderada de diabetes entre adultos mexiquenses fue del $12.28 \%$ en hombres y $10.26 \%$ en mujeres. En tanto, la media del IMC fue mayor para las mujeres, pero el comportamiento es contrario respecto de la $\mathrm{CC}$ con un mayor perímetro para los hombres. La media de la edad fue 43.7 años en hombres y 42.2 en mujeres. Respecto de los antecedentes parentales de diabetes, el $36.4 \%$ de los hombres y $42.5 \%$ de las mujeres reportó historial. 
Tabla 1. Estadísticos descriptivos de las variables en análisis, por sexo.

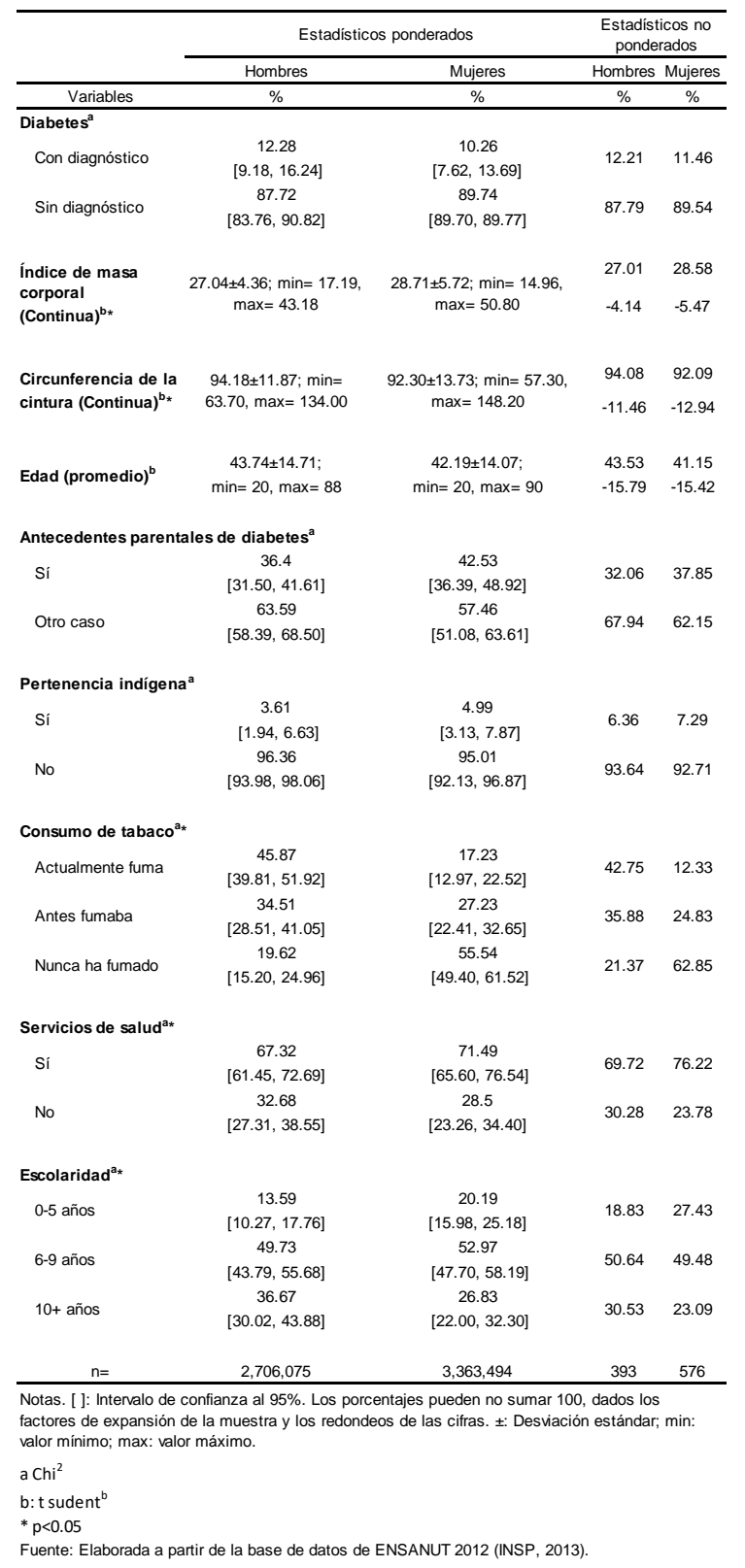

El gráfico 1 ilustra las curvas ROC para el IMC y la $\mathrm{CC}$ por sexo. Se presentan las pruebas de diferencias entre las $\mathrm{ABC}$, así como los valores de sensibilidad (\%) y especificidad (\%) de cada indicador. En el caso de los hombres, se estimó una mayor $\mathrm{ABC}$ para la $\mathrm{CC}(\mathrm{IMC}=0.562, \mathrm{CC}=0.682)$ y la diferencia en las $\mathrm{ABC}$ de los hombres fue significativa $(\chi 2=26.84, \mathrm{p}=$
0.000). En el caso de las mujeres, los resultados fueron consistentes con los de los hombres (IMC= $0.573, \quad \mathrm{CC}=0.652), \quad$ siendo estadísticamente significativa la diferencia entre las $\mathrm{ABC}\left(\chi^{2}=12.33\right.$, $\mathrm{p}=0.000)$. Estos resultados permiten concluir que la CC de la cintura sería un mejor predictor de la diabetes que el IMC tanto en hombres como en mujeres.

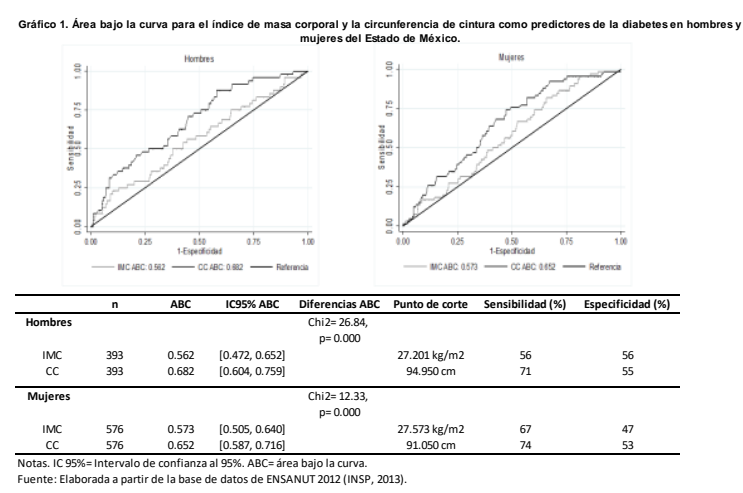

En la tabla 2 se resumen los resultados de los modelos de regresión logística para hombres. Se encontró que tener una $\mathrm{CC} \geq 94.95 \mathrm{~cm}$, multiplica por 2.3 veces la propensión a tener diabetes (RM sin ajustar $=2.98 ; \mathrm{RM}$ ajustado= 2.35). Sin embargo, en el caso del IMC no se halló significancia del punto de corte definido en las estimaciones de las curvas ROC. Además, se estimó que un incremento de un año en la edad representa un aumento de cerca del $5.0 \%$ en la propensión a presentar diabetes (RM ajustado $=1.05$ para IMC), un resultado consistente para el IMC y la CC. Asimismo, es importante notar que, aun controlando por el efecto del IMC o de la $\mathrm{CC}$ y las de control, los hombres con antecedentes parentales de diabetes tenían momios entre 2.6 y 2.7 veces los momios de tener la enfermedad que las personas sin antecedentes $(\mathrm{RM}$ ajustado $=2.73$ para IMC, y RM ajustado= 2.57 para CC). 


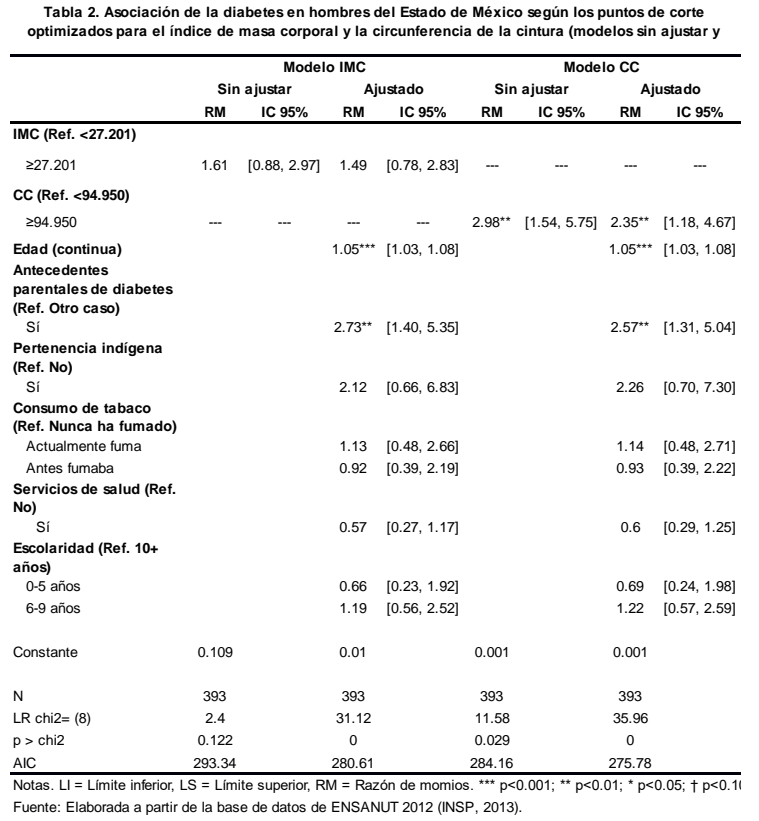

En la tabla 3 se presentan los resultados de los modelos de regresión logística para mujeres. Entonces, se halló significancia para el punto de corte del IMC $\geq 27.573 \mathrm{~kg} / \mathrm{m} 2$ el cual indica que las mujeres con este IMC tienen una probabilidad $81.0 \%$ mayor de tener diabetes que aquellas con un IMC menor, sin embargo, solo el modelo sin ajustar fue significativo $(\mathrm{RM}$ ajustado= 1.81). En el caso de la CC los resultados fueron consistentes dado que para las mujeres tener una $\mathrm{CC} \geq 91.050 \mathrm{~cm}$, multiplicaba por 2.6 veces la propensión a tener diabetes que la de aquellas mujeres con una $\mathrm{CC}<91.050 \mathrm{~cm}$ (RM sin ajustar $=3.22 ; \mathrm{RM}$ ajustado $=2.60$ ). Además, se calculó que un incremento de un año en la edad representa un aumento de cerca del $6.0 \%$ en la propensión a presentar diabetes (RM ajustado= 1.06). En cuanto a los antecedentes parentales de diabetes, las personas con esta condición tenían momios cercanos a 3.2 veces los momios de tener la enfermedad que las mujeres sin historial paterno de diabetes (RM ajustado= 3.22 para IMC y RM ajustado= 3.22 para $\mathrm{CC}$ ). Además, y si bien los resultados son marginalmente significativos, es notorio que, los momios para la diabetes de las mujeres con afiliación a servicios de salud fueron 2.1 veces los momios de aquellas sin afiliación (RM ajustado $=2.15$ para IMC y $\mathrm{RM}$ ajustado $=2.15$ para $\mathrm{CC})$.

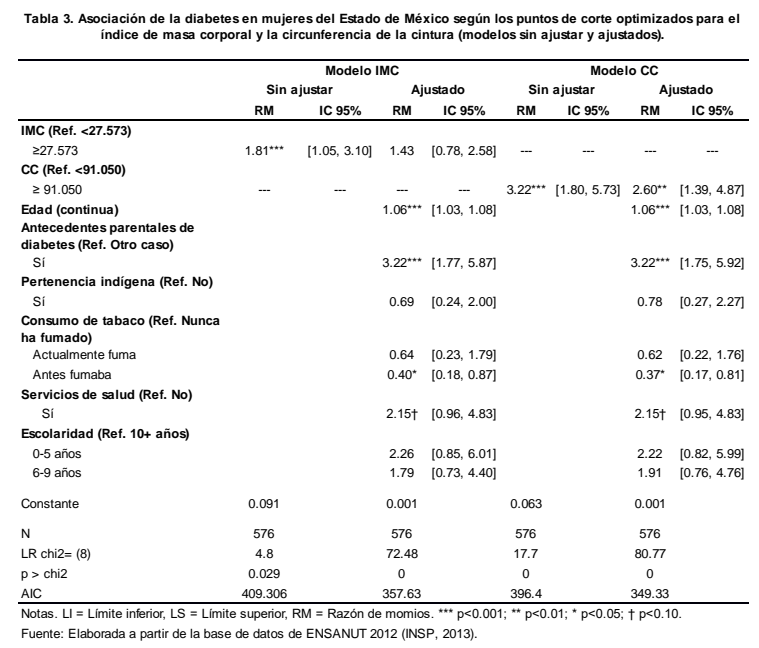

\section{Discusión}

En este estudio se encontró que, en población adulta del Estado de México, la CC es un mejor predictor de la diabetes que el IMC. Este efecto se mantuvo constante aun después de ajustarlo por las variables propuestas: edad, antecedentes parentales de diabetes, pertenencia indígena, consumo de tabaco, escolaridad y afiliación a servicios de salud. En el caso del IMC solo el modelo sin ajustar para las mujeres fue significativo, es decir, aquellas con un IMC $\geq 27.573 \mathrm{~kg} / \mathrm{m} 2$ tenía una mayor probabilidad de diabetes DM. En caso contrario, los resultados del indicador $\mathrm{CC}$ exponen que, los hombres con una $\mathrm{CC} \geq 94.950 \mathrm{~cm}$ y las mujeres con una $\mathrm{CC} \geq 91.050 \mathrm{~cm}$ tienen mayores probabilidades de presentar diabetes. Respecto de estos resultados, la discusión se amplía ya que en ambos casos estos puntos de corte son diferentes a los propuestos por la OMS (2000). En el caso de las mujeres se considera que una $\mathrm{CC} \geq 88 \mathrm{~cm}$ incrementa el riesgo de tener diabetes y este punto es menor en aproximadamente $3 \mathrm{~cm}$ que el obtenido aquí $(91.050 \mathrm{~cm})$. Por el contrario, en el caso de los hombres, el punto de corte sugerido por la OMS es mayor en 7 centímetros ( $\geq 102 \mathrm{~cm}$ ) que el punto aquí estimado en $94.950 \mathrm{~cm}$. Incluso este punto de corte es mayor que el considerado por la Secretaría de Salud de México $(\geq 90 \mathrm{~cm})$ (Secretaría de Salud, 2005).

Los resultados anteriores son complejos y podrían entenderse como reflejo de la heterogeneidad presente en las poblaciones, pues existen discusiones en torno la variación de los puntos de corte óptimos 
que se asociarían con diferencias biológicas o por factores como el método empleado para su identificación (Rojas-Martínez et al., 2012). Además, sobre la discusión de la capacidad predictiva del indicador $\mathrm{CC}$ en la diabetes se han encontrado diferencias relacionadas con aspectos como el uso de datos longitudinales o transversales (Qiao y Nyamdorj, 2010) o incluso por las diferencias en la composición corporal propias de la edad (Chen et al., 2009; Rojas-Martínez, AguilarSalinas y Jiménez-Corona, 2012). Incluso, estas diferencias se encuentran respaldadas por las diversas recomendaciones de distintos organismos que se relacionan en parte con las composiciones raciales de las poblaciones (Min y Stephens, 2015), pues, por ejemplo, los puntos de corte de la OMS fueron desarrollados en poblaciones con una fuerte componente caucásica. Elementos como los anteriores confirman entonces, la necesidad de explorar las diferencias en los puntos de corte de la CC para la detección de diabetes entre diferentes poblaciones como las planteadas aquí para el Estado de México.

Otro resultado destacable de esta investigación es el que relaciona los antecedentes parentales de diabetes con las probabilidades de tener esta enfermedad, incluso ajustando por la CC o el IMC y las demás variables de control. Específicamente, aquellos adultos del Estado de México que informaron tener algún antecedente parental de diabetes, la propensión a presentar esta enfermedad se multiplicó por entre 2.5 y 3.2 veces tanto para el IMC como para la CC. De manera similar, Pinto y Beltrán-Sánchez (2015) encontraron que, ante la presencia de antecedentes parentales, se multiplicaba por dos la probabilidad de presentar diabetes.

Por otro lado, aunque no se encontró que fueran estadísticamente relevantes los efectos de la afiliación a algún servicio de salud, los datos indicaron que la condición de contar con afiliación multiplica por 2.1 veces la propensión a presentar diabetes entre las mujeres, un resultado consistente con los de otras investigaciones en las que se estimó que la afiliación a servicios de salud aumenta la probabilidad de tener reporte positivo de diabetes. No obstante, se ha hipotetizado que este resultado puede estar influido por el hecho de que las mujeres suelen utilizar en mayor medida los servicios diagnósticos y de atención a la salud (Salinas, 2015).
Este trabajo presenta como fortalezas el utilizar una muestra representativa a nivel estatal para analizar la asociación de la diabetes y la obesidad. Seguido de lo anterior, en esta investigación se empleó antropometría en la evaluación de la CC y el IMC lo cual disminuye la probabilidad de subestimar tales indicadores ( $\mathrm{Ng}, 2019)$. Adicionalmente, esta investigación analizó datos analizados, de una muestra representativa a nivel estatal que corresponden a la ENSANUT 2012, y aunque datan de hace ya ocho años, ésta es la única encuesta disponible con representatividad de la población mexiquense, específicamente en el ámbito de los indicadores antropométricos para el IMC y la CC, pues la ENSANUT 2018 no es representativa en este rubro a nivel entidad federativa (INSP, 2019b).

En tanto limitaciones de esta investigación, pueden señalarse: 1. Aunque el autoreporte de diabetes ha mostrado ser consistente, este podría relacionarse con determinados sesgos en la declaración; 2 . Si bien existe evidencia de que factores como el tiempo de evolución de la obesidad y la edad en la que se incrementó el peso corporal inciden en el riesgo de desarrollar diabetes (González-Villalpando et al., 2004), estas asociaciones quedaron fuera del alcance de esta investigación; 3. La pertenencia indígena podría estar limitada dado el criterio definitorio usado; 4. Al igual que cualquier investigación no se descarta la presencia de heterogeneidad no observada que pudo influir en resultados como la no significancia del IMC al ajustar las regresiones. Sin embargo, la ausencia de variables como, por ejemplo, la actividad física (AF) se debió a que en la ENSANUT 2012, el instrumento de evaluación de la AF se aplicó a una submuestra (19-69 años) (Gutiérrez et al., 2012), lo que disminuía ampliamente los tamaños muestrales aquí analizados.

\section{Conclusiones}

a pesar de encontrar diferencias con los puntos de corte establecidos por la OMS (2000), esta investigación expone que en la población adulta del Estado de México el indicador de la $\mathrm{CC}$ es un buen predictor de la probabilidad de presentar diabetes, incluso más consistente que el IMC. Estos resultados son fundamentales en un contexto en el que las pruebas diagnósticas de diabetes entre la población adulta (20-79 años de edad) del Estado de México fue del $23.0 \%$, y solo el $10.6 \%$ declaró haber recibido 
tratamiento para la enfermedad (INSP, 2013). Además, los resultados aquí obtenidos permiten sugerir la utilización de la CC como un indicador cuya facilidad de obtención permitiría identificar de manera rápida a las personas en riesgo de tener diabetes.

\section{Bibliografía}

Albrecht, S. S., Barquera, S. y Popkin, B. M. (2014). Exploring secular changes in the association between BMI and waist circumference in Mexican-origin and white women: A comparison of Mexico and the United States. American Journal of Human Biology, 26, 627-634. DOI: 10.1002/ajhb.22573

Al-Goblan, A. S., Al-Alfi, M. A. y Khan, M. Z. (2014). Mechanism linking diabetes mellitus and obesity. Diabetes, Metabolic Syndrome and Obesity: Targets and Therapy, 7, 587-591. https://www.ncbi.nlm.nih.gov/pmc/articles/PMC 4259868/

American Diabetes Association. (2020a). Introduction: Standards of medical care in diabetes-2020. Diabetes Care, 43(S1), S1-S2. https://doi.org/10.2337/dc20-SINT

American Diabetes Association. (2020b). Classification and diagnosis of diabetes: Standards of medical care in diabetes-2020. Diabetes Care, 43(S1), S14-S31. https://doi.org/10.2337/dc20-S002

Aucott, L. S. (2008). Influences of weight lost son long-term diabetes outcomes. Proceedings of the Nutrition Society, 67, 54-59. https://doi.org/10.1017/S0029665108006022

Barquera, S., Campos-Nonato, I., HernándezBarrera, L., Pedroza-Tobías, A. y RiveraDommarco, J. A. (2013). Prevalencia de obesidad en adultos mexicanos, ENSANUT 2012. Salud Pública de México, 55(sup. 2), S151-S160.

Beltrán-Sánchez, H., Drumond-Andrade, F. C. y Riosmena, F. (2015). Contribution of socioeconomic factors and health care access to the awareness and treatment of diabetes and hypertension among older Mexican adults. Salud
Pública de México, 57(1), 317-322. http://dx.doi.org/10.21149/spm.v57s1.7584

Bush, T., Lovejoy, J. C., Deprey, M. y Carpenter, K. M. (2016). The effect of tobacco cessation on weight gain, obesity, and diabetes risk. Obesity, 24 , 1834-1841. https://www.ncbi.nlm.nih.gov/pmc/articles/PMC 5004778/

Cederberg, H., Stancakova, A., Kuusisto, J., Laakso, M. y Smith, U. (2015). Family history of type 2 diabetes increases the risk of both obesity and its complications: Is type 2 diabetes a disease of inappropriate lipid storage? Journal of Internal Medicine, 277(5), 540-551. https://doi.org/10.1111/joim.12289

Cerhan, J. R., Moore, S. C., Jacobs, E. J., Kitahara, C. M., Rosenberg, P.S., Adami, H-O., Ebbert, J. O... Berrington de Gonzalez, A. (2014). A pooled analysis of waist circumference and mortality in 650,000 adults. Mayo Clinic Proceedings, $\quad$ 89(3), 335-345. https://doi.org/10.1016/j.mayocp.2013.11.011

Chen, C-C., Wang, W-S., Chang, H-Y., Liu, J-S. y Chen, Y-J. (2009). Heterogeneity of body mass index, waist circumference, and waist-to-hip ratio in predicting obesity-related metabolic disorders for Taiwanese aged 35. Clinical Nutrition, 28, 543-548.

https://linkinghub.elsevier.com/retrieve/pii/S026 1-5614(09)00091-0

Chooi, Y. C., Ding, C. y Magkos, F. (2019). The epidemiology of obesity. Metabolism, 92, 6-10. https://pubmed.ncbi.nlm.nih.gov/30253139-theepidemiology-of-obesity/

Cleves, M. (2002). From the help desk: Comparing areas under receiver operating characteristic curves from two or more probit or logit models. The Stata Journal, 2(3), 301-313. https://www.statajournal.com/sjpdf.html?articlenum=st0023

Cohen, A. K., Rai, M., Rehkopf, D. H. y Abrams, B. (2013). Educational attainment and obesity; a systematic review. Obesity Reviews, 14(12), 9891005. 
https://www.ncbi.nlm.nih.gov/pmc/articles/PMC 3902051/

Consejo Nacional de Población. (2018). Proyecciones de población de México y de entidades federativas 2016-2050. Disponible en: Instituto Nacional de Estadística y Geografía. (2020). Encuesta Intercensal. Tabulados. Disponible en: https://www.inegi.org.mx/programas/intercensal /2015/\#

den Braver, N. R., Lakerveld, J., Rutters, F., Schoonmade, L. J., Brug, J. y Beulesn, J. W. (2018). Built environmental characteristics and diabetes: A systematic review and meta-analysis. Diabetes: Prevention, management and treatment, $16(1), \quad 12-37$. https://doi.org/10.1186/s12916-017-0997.

Estampador, A. C. y Franks, P. W. (2018). Precision medicine in obesity and type 2 diabetes: the relevance of early-life exposures. Clinical Chemistry, 64, 1-12. DOI: 10.1373/clinchem.2017.273540

Feller, S., Boeing, H. y Pischon, T. (2010). Body mass index, waist circumference, and the risk of Type 2 diabetes mellitus. Implications for Routine Clinical Practice. Deutsches Arzbeteblatt International, 107(26), 470-476. https://www.ncbi.nlm.nih.gov/pmc/articles/PMC 2905837/

Gatineau, M., Hancock, C., Holman, N., Outhwaite, H., Oldridge, L., Christie, A. y Ells, L. (2014). Adult obesity and type 2 diabetes. Public Health England. Disponible en: https://www.google.com/url?sa=t\&rct=j\&q=\&es $\mathrm{rc}=\mathrm{s} \&$ source $=$ web\&cd=3\&ved=2ahUKEwjs64 ciKDnAhVQRa0KHVi7Ck0QFjACegQIDBAE \&url=https $\% 3 \mathrm{~A} \% 2 \mathrm{~F} \% 2 \mathrm{Fassets}$.publishing.servi ce.gov.uk\%2Fgovernment $\% 2$ Fuploads $\% 2$ Fsyste m\%2Fuploads\%2Fattachment_data\%2Ffile $\% 2 \mathrm{~F}$ 338934\%2FAdult_obesity_and_type_2_diabetes _.pdf\&usg=AOvVaw06-YRVDAqismY2epXqM_8

Gómez-Ambrosi, J., Silva, C., Galofré, J., Escalada, J., Santos, S., Gil, M., Valenti, V... Frühbeck, G. (2011). Body adiposity and type 2 diabetes:
Increased risk with a high body fat percentage even having a normal BMI. Obesity, 19(7), 14391444.

González-Villalpando, C., Dávila-Cervantes, C. A., Zamora-Macorra, M., Trejo-Valdivia, B. y González-Villalpando, M. E. (2014). Risk factors associated to diabetes in Mexican population and phenotype of the individuals who will convert to diabetes. Salud Pública de México, 56(4), 317322. http://dx.doi.org/10.21149/spm.v56i4.7351

Gutiérrez, J. P., Rivera-Dommarco, J., ShamahLevy, T., Villalpando-Hernández, S., Franco, A., Cuevas-Nasu, L., Romero-Martínez, M. y Hernández-Ávila, M. (2012). Encuesta Nacional de Salud y Nutrición 2012: Resultados nacionales. Cuernavaca, México: Instituto Nacional de Salud Pública. Disponible en https://ensanut.insp.mx/encuestas/ensanut2012/i nformes.php

Haffner, S. (1998). Epidemiology of type 2 diabetes: Risk factors. Diabetes Care, 21(3), 3-6. http://dx.doi.org/10.2337/diacare.21.3.c3

Hernández-Ávila, M., Gutiérrez, J. y ReynosoNoverón, N. (2013). Diabetes mellitus en México: El estado de la epidemia. Salud Pública de México, 55(2), 129-136. http://dx.doi.org/10.21149/spm.v55s2.5108

Hilding, A., Eriksson, A-K., Agardh, E. E., Grill, V., Ahlbom, A., Efendic, S. y Östenson, C-G. (2006). The impact of family history of diabetes and lifestyle factors on abnormal glucose regulation in middle-aged Swedish men and women. Diabetologia, $\quad 49(11), \quad$ 2589-2598. https://doi.org/10.1007/s00125-006-0402-5

Huebschmann, A. G., Huxley, R. R., Kohrt, W. M., Zeitler, P., Regensteiner, J. G. y Reusch, J. E. B. (2019). Sex differences in the burden of type 2 diabetes and cardiovascular risk across the life course. Diabetologia, 62(10), 1761-1772. https://link.springer.com/article/10.1007\%2Fs00 125-019-4939-5

Instituto Nacional de Salud Pública. (2013). Encuesta Nacional de Salud y Nutrición 2012: Resultados por entidad federativa, Estado de 
México. Cuernavaca, México: Instituto Nacional de Salud Pública. Disponible en https://ensanut.insp.mx/encuestas/ensanut2012/i nformes.php

Instituto Nacional de Salud Pública. (2016). Encuesta Nacional de Salud y Nutrición de Medio Camino 2016: Informe final de resultados. Cuernavaca, México: Instituto Nacional de Salud Pública. Disponible en https://www.gob.mx/cms/uploads/attachment/fil e/209093/ENSANUT.pdf

Instituto Nacional de Salud Pública. (2019a). Encuesta Nacional de Salud y Nutrición 2018. Presentación de resultados. Cuernavaca, México: Instituto Nacional de Salud Pública. Disponible en https://ensanut.insp.mx/encuestas/ensanut2018/i nformes.php

Instituto Nacional de Salud Pública. (2019b). Encuesta Nacional de Salud y Nutrición 2018. Diseño Muestral. Cuernavaca, México: Instituto Nacional de Salud Pública. Disponible en https://ensanut.insp.mx/encuestas/ensanut2018/i nformes.php

Janssen, I., Katzmarzyk, P. T. y Ross, R. (2004). Waist circumference and not body mass index explains obesity-related health risk. The American Journal of Clinical Nutrition, 79(3), 379-384.

Jarolimova, J., Tagoni, J. y Stern, T. (2013). Obesity: Its epidemiology, comorbidities, and management. Primary Care Companion for CNS Disorders, $\quad 15(5), \quad 1475$. http://dx.doi.org/10.4088/PCC.12f01475

Kahn, S. E., Hull, R. L. y Utzschneider, K. M. (2006). Mechanisms linking obesity to insulin resistance and type 2 diabetes. Nature, 444(7121), 840-846. DOI: $10.1038 /$ nature05482

Kanter, R. y Caballero, B. (2012). Global gender disparities in obesity: a review. Advances in Nutrition, 3(4), 491-498. https://www.ncbi.nlm.nih.gov/pmc/articles/PMC 3649717/
Katzmarzyk, P. T., Bray, G. A., Greenway, F. L., Johnsson, W. D., Newton, R. L., Ravussin, E... Bouchard, C. (2011). Ethnic-specific BMI and waist circumference thresholds. Obesity, 19(6), 1272-1278.

Kim, Y-H., Kim, S. M., Han, K-D., Jung., J-H., Lee, S-S., Oh, S. W., Park, H. S... Yoo, S. J. (2019). Waist Circumference and All-Cause Mortality Independent of Body Mass Index in Korean Population from the National Health Insurance Health Checkup 2009-2015. Journal of Clinical Medicine, $\quad 8, \quad 72-82$. https://www.ncbi.nlm.nih.gov/pmc/articles/PMC 6352259/

Kirkman, M. S., Briscoe, V. J., Clark, N., Florez, H., Haas, L. B., Halter, J. B... Swift, C. (2012). Diabetes in older adults. Diabetes Care, 35(12), 2650-2665. https://doi.org/10.2337/dc12-1801

Kutbi, H. A., Mosli, H. H., Alhasan, A. H. y Mosli, R. H. (2018). Diabetes knowledge and its association with the weight status among residents of Jeddah City, Saudi Arabia. Nutrition \& Diabetes, 8, 48-56. https://doi.org/10.1038/s41387-018-0055-8

Liu, Y., Tong, G., Tong, W., Lu, L. y Qin, X. (2011). Can body mass index, waist circumference, waist-hip ratio and waist-height ratio predict the presence of multiple metabolic risk factors in Chinese subjects? BMC Public Health, 11, 35-45. http://www.biomedcentral.com/14712458/11/35

Liu, X. (2012). Classification accuracy and cut point selection. Statistics in Medicine, 31(23), 26762686.

Min, T. y Stephens, J. W. (2015). Targeting abdominal obesity in diabetes. Diabetes Management, 5(4), 301-309. Doi: 10.2217/DMT.15.14

Ng, C. (2019). Biases in self-reported height and weight measurements and their effects on modeling health outcomes. SSM - Population Health, 7, 1-11. https://www.sciencedirect.com/science/article/pi i/S235282731830346X 
Olaiz-Fernández, G., Rojas, R., Aguilar-Salinas, C. A., Rauda, J. y Villalpando, S. (2007). Diabetes mellitus en adultos mexicanos: Resultados de la Encuesta Nacional de Salud 2000. Salud Pública de México, 49(3), 331-337.

Organización Mundial de la Salud [OMS] (2000). Obesity: Preventing and managing the global epidemic of obesity. Report of a WHO Consultation (WHO Technical Report Series 894). Disponible en: https://www.who.int/nutrition/publications/obesi ty/WHO_TRS_894/en/

Organización Mundial de la Salud [OMS] (2018). Diabetes. Disponible en: https://www.who.int/news-room/factsheets/detail/diabetes

Pinto, G. y Beltrán-Sánchez, H. (2015). Prospective study of the link between overweight/obesity and diabetes incidence among Mexican older adults: 2001-2012. Salud Pública de México, 57(1), 15 21. http://dx.doi.org/10.21149/spm.v57s1.7585

Qiao, Q. y Nyamdorj, R. (2010). Is the Association of type II diabetes with waist circumference or waist-to-hip ratio stronger tan that with body mass index? European Journal of Clinical Nutrition, 64, 30-34. doi:10.1038/ejcn.2009.93

Rojas-Martínez, R., Aguilar-Salinas, C. A. y Jiménez-Corona, A. (2012). Optimal cut-off points fir the detection of undiagnosed type 2 diabetes, hypertension and metabolic síndrome in Mexican adults. Salud Pública de México, 54(1), 13-19.

Rojas-Martínez, R., Aguilar-Salinas, C., ZárateRojas, E., Villalpando, S. y Barrientos-Gutiérrez, T. (2018). Prevalencia de diabetes por diagnóstico médico previo en México. Salud Pública de México, 60(3), 224-232. https://doi.org/10.21149/8566

Romero-Martínez, M., Shamah-Levy, T., FrancoNuñez, A., Villalpando, S., Cuevas-Nasú, L., Gutiérrez, S. y Rivera-Dommarco, J. (2013). Encuesta Nacional de Salud y Nutrición 2012: Diseño y cobertura. Salud Pública de México, 55(2), 332-340.
Romero-Martínez, M., Shamah-Levy, T., CuevasNasú, L., Méndez Gómez-Humarán, I., GaonaPineda, B., Gómez-Acosta, L. (2017). Diseño metodológico de la Encuesta Nacional de Salud y Nutrición de Medio Camino 2016. Salud Pública de México, 59, 299-305. http://doi.org/10.21149/8593

Rull, J. A., Aguilar-Salinas, C. A., Rojas, R., RíosTorres, J. M., Gómez-Pérez, F. J. y Olaiz, G. (2005). Epidemiology of type 2 diabetes in Mexico. Archives of Medical Research, 36(1), 188-196.

http://doi.org/10.1016/j.arcmed.2005.01.006

Salinas, J. J. (2015). Preventive health screening utilization in older Mexicans before and after healthcare reform. Salud Pública de México, 57(1), $\mathrm{S} 70-\mathrm{S} 78$. http://dx.doi.org/10.21149/spm.v57s1.7592

Secretaria de Salud. (2005). Menor circunferencia abdominal, mejor salud. Reporte 740. México: Secretaría de Salud. Disponible en: http://www.salud.gob.mx/ssa_app/noticias/datos /2005-12-31_1914.html

Secretaría de Salud. (2020). Anuarios de morbilidad. 20 principales causas de enfermedad estatal por grupo de edad. Disponible en http://www.epidemiologia.salud.gob.mx/anuario /html/principales_estatal_grupo.html

Shang, X., Li, J., Tao, Q., Li, J., Li, X., Zhang, L., Liu, X... Yang, Y. (2013). Educational level, obesity and incidence of diabetes among Chinese adult men and women aged 18-59 years old: an 11-year follow-up study. PLoS ONE, 8(6), e66479.

https://doi.org/10.1371/journal.pone.0066479

Sistema Nacional de Vigilancia Epidemiológica / Dirección General de Epidemiología/Secretaría de Salud [SINAVE/DGE/SALUD]. (2020). Defunciones por año de registro. Disponible en: http://sinaiscap.salud.gob.mx:8080/DGIS/

Sosenko, J. M., Kato, M., Soto, R. y Goldberg, R. B. (1993). A comparison of adiposity measures for screening non-insulin dependent diabetes mellitus. International Journal of Obesity and Related Metabolic Disorders, 17(8), 441-444. 
StataCorp. (2016). Stata statistical software: Release 14. College Station, Texas: StataCorp LLC.

Steele, C. J., Schöttker, B., Marshall, A. H., Kouvonen, A., O’Doherty, M. G., Mons, U., Saum, K-U... Kee, F. 82017). Education achievement and type 2 diabetes - what mediates the relationship in older adults? Data from the ESTHER study: a population-based cohort Study. BMJ Open, 7, e013569. doi:10.1136/bmjopen-2016-013569

Vázquez, G., Duval, S., Jacobs, D. R. y Silventoinen, K. (2007). Comparison of body mass index, waist circumference, and waist/hip ratio in predicting incident diabetes: a meta-analysis. Epidemiologic Reviews, 29, 115-128.

Ye, J. (2013). Mechanisms of insulin resistance in obesity. Frontiers of Medicine, 7(1), 14-24. doi: 10.1007/s11684-013-0262-6

Yuan, X., Liu, T., Wu, L., Zou, Z. Y. y Li, C. (2015). Validity of self-reported diabetes among middleaged and older Chinese adults: The China Health and Retirement Longitudinal Study. BMJ Open, 5, e006633. http://dx.doi.org/10.1136/bmjopen2014-006633

Yusuf, S., Hawken, S., Ounpuu, S., Bautista, L., Franzosi, M. G., Commerford, P., Lang, C. C... Anand, S. S. (2005). Obesity and the risk of myocardial infarction in 27000 participants from 52 countries: a case-control study. Lancet, 366, 1640-1649. 